\title{
Constraining statistical-model parameters using fusion and spallation reactions
}

\author{
Davide Mancusi $^{1}$, Robert J. Charity ${ }^{2}$, and Joseph Cugnon ${ }^{1}$ \\ 1 University of Liège, AGO Department, allée du 6 Août 17, bât. B5, B-4000 Liège 1, Belgium \\ 2 Department of Chemistry, Washington University, St. Louis, Missouri 63130, USA
}

\begin{abstract}
The de-excitation of compound nuclei has been successfully described for several decades by means of statistical models. However, such models involve a large number of free parameters and ingredients that are often underconstrained by experimental data. We show how the degeneracy of the model ingredients can be partially lifted by studying different entrance channels for de-excitation, which populate different regions of the parameter space of the compound nucleus. Fusion reactions, in particular, play an important role in this strategy because they fix three out of four of the compound-nucleus parameters (mass, charge and total excitation energy). The present work focuses on fission and intermediate-mass-fragment emission cross sections. We prove how equivalent parameter sets for fusion-fission reactions can be resolved using another entrance channel, namely spallation reactions. Intermediate-mass-fragment emission can be constrained in a similar way. An interpretation of the best-fit IMF barriers in terms of the Wigner energies of the nascent fragments is discussed.
\end{abstract}

\section{Introduction}

New motivation for the study of nuclear de-excitation has recently emerged from novel application fields such as radioprotection in space [1], hadrontherapy [2] and the design of accelerator-driven systems [3]. All these applications involve the de-excitation of highly excited nuclei; this phenomenon is qualitatively well-understood and usually described by statistical models. However, such models contain a great deal of free parameters and assumptions (or, more generally, ingredients), that are often underconstrained by the available experimental data. Quantitatively accurate predictions usually require some tuning of the model parameters.

The fusion entrance channel is a particularly valuable tool to explore the sensitivity of the de-excitation model to the compound-nucleus parameters (mass, charge, excitation energy and spin); if the cross sections for incomplete fusion and pre-equilibrium emission are small with respect to the fusion cross section for a given projectiletarget combination, the compound nucleus can essentially be regarded as having a fixed mass, charge and total excitation energy (intrinsic plus collective), thereby fixing three of the four parameters that describe it. The requirement of complete fusion, however, puts an upper limit on the energy of the projectile and, thus, on the excitation energies that can be studied with this method. Because of this and other similar limitations on the entrance channel, one is actually able to construct different parameter sets that can describe the same experimental data to a similar degree of accuracy; in this sense, statistical de-excitation models contain partly degenerate ingredients, and that limits their predictive power.
On the other hand, part of the degeneracy can be removed by performing simultaneous fits to heterogeneous data sets. For example, one can try to explore diverse regions of the compound-nucleus parameter space by studying different reaction entrance channels. The present work applies this idea to the study of fission and emission of intermediate-mass fragments (IMFs) by combining the fusion and the spallation entrance channels. The goal is to put more stringent constraints on the de-excitation-model parameters than those that would be obtained from the separate study of fusion- and spallation-induced de-excitation chains. Spallation and fusion complement each other almost ideally: while the latter extensively probes the sensitivity of de-excitation to the compound-nucleus spin, but is limited in excitation energy by the requirement of complete fusion, the former tends to produce nuclei with larger excitation energies and small spins [4]. Relativistic heavyion collisions could also be used for a similar purpose, e.g. as recently demonstrated by Schmitt et al. [5].

\section{Tools}

This work focuses on the GEMINI++ nuclear de-excitation model [6], which was originally developed to describe the formation of fragments in heavy-ion-induced reactions. Since these reactions are typically characterised by large spins of the compound nucleus, GEMINI++ was designed to accurately model changes in orbital and intrinsic angular momentum of the de-excitation products. This is particularly important for the study of fission and IMF emission, which are quite sensitive to the spin of the mother nucleus.

For nuclides above the Businaro-Gallone point, the ridge of conditional saddle points as a function of the asymmetry 


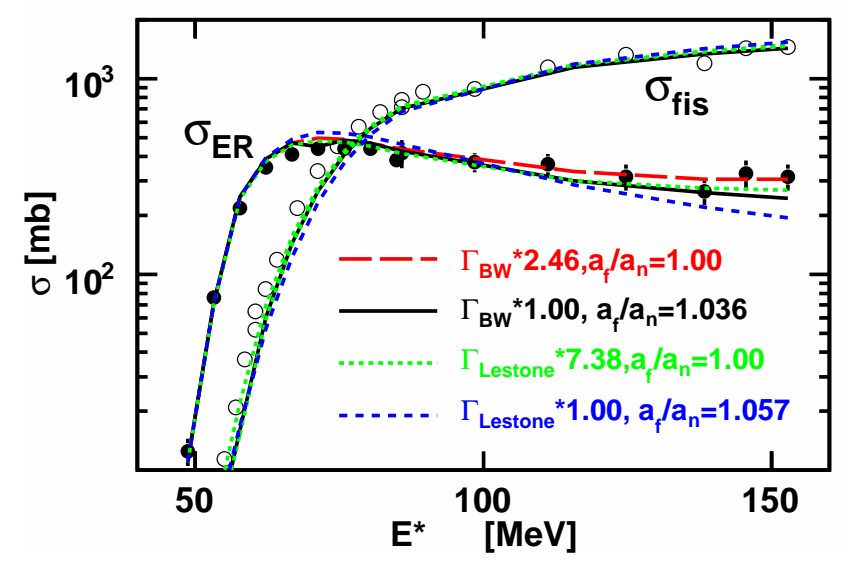

Figure 1. Experimental $[7,8]$ and calculated GEMINI++ predictions for evaporation-residue and fission excitation functions for the ${ }^{19} \mathrm{~F}+{ }^{181}$ Ta reaction.

of the split exhibits a minimum around symmetric splitting and two local maxima on either side (apart from local variations due to structure effects). For such systems, GEMINI++ adopts a global description of fission. The statistical width of the process is computed using a BohrWheeler-type formalism, with barriers taken from Sierk's finite-range calculations $[9,10]$. In addition, several corrections are possible within the framework of the model: (a) different level-density parameters at the saddle point and in the ground state, (b) a constant shift of the Sierk barrier heights, (c) overall scaling of the fission width, and (d) explicit treatment of the tilting degree of freedom at saddle [11]. This establishes the free ingredients of our fission model. The scission mass and charge distributions are taken from Rusanov et al.'s systematics [12].

GEMINI++ also considers the emission of fragments with $3<A<A_{\mathrm{IMF}}$, where $A_{\mathrm{IMF}}$ is the fragment mass corresponding to the first maximum in the ridge of conditional saddle points. This process is also described by a transition-state formalism [13], with explicitly singled-out mass- and charge-asymmetry degrees of freedom at saddle. Given the formal similarity, the IMF-emission model includes the same free ingredients as the fission model. Finally, the emission of nucleons and light clusters $(A \leq 3)$ is described by the Hauser-Feshbach evaporation formalism [14].

Besides the de-excitation model, the proposed task requires models for the reaction entrance channels. For fusion, we limit our study to incident energies lower than about $10 \mathrm{AMeV}$, where incomplete fusion and pre-equilibrium should be negligible; thus, we only need to specify the spin distribution of the compound nucleus. We assume the following roughly triangular shape:

$$
\sigma_{\text {fus }}(J)=\pi \hbar^{2}(2 J+1)\left[1+\exp \left(\frac{J-J_{0}}{\delta J}\right)\right]^{-1},
$$

where $J_{0}$ determines the maximum spin value and $\delta J$ plays the role of a smooth cutoff. The $J_{0}$ parameter is fixed from

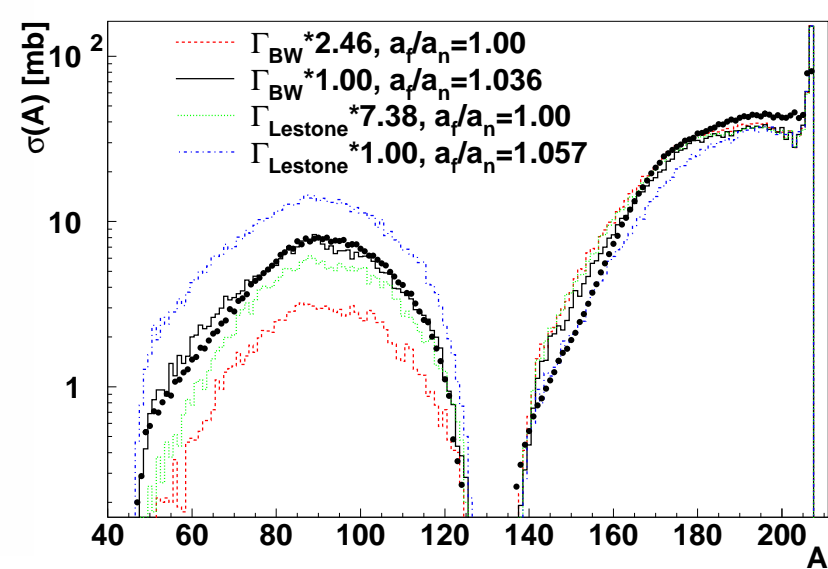

Figure 2. Experimental [15] and calculated residual mass distributions for the $1-\mathrm{GeV} p+{ }^{208} \mathrm{~Pb}$ reaction.

the total fusion cross section

$$
\sigma_{\text {fus }}=\sum_{J=0}^{\infty} \sigma_{\text {fus }}(J),
$$

while $\delta J$ is set to values from 3 to $10 \hbar$, with the larger values associated with the heavier projectiles. For the reactions for which we present IMF data, the fusion cross sections have not been measured and the Bass model [16] is used to calculate both the cross sections and maximum spin values.

The entrance channel for spallation reactions is described by the Liège Intranuclear-Cascade model (INCL) [17]. In this framework, the high-energy incident nucleon initiates an avalanche of binary nucleon-nucleon collisions within the target nucleus, which can lead to the emission of a few nucleons and possibly pions. The cascade is stopped when the cascade remnant shows signs of thermalisation. This provides the entry point for the GEMINI++ de-excitation chain. A more comprehensive description of the latest INCL developments has been recently published [18]. One should stress here that the INCL model does have internal parameters, but they have been either taken from known phenomenology (e.g. the parameters describing nuclear density distributions) or fixed once and for all (e.g. the parameters connected with the description of Pauli collision blocking). Thus, the present work only focuses on the adjustment of the GEMINI++ side of the reaction model.

\section{Results and discussion}

We first discuss fusion-fission and spallation-fission calculations for compound nuclei of similar mass and charge. Figure 1 shows the result of four fits to fusion-fission data: here $\Gamma_{\mathrm{BW}}$ and $\Gamma_{\text {Lestone }}$ indicate calculations performed without or with Lestone's tilting correction, respectively; a global scaling factor is applied in some parameter sets; and $a_{\mathrm{f}} / a_{\mathrm{n}}$ represents the ratio of the level-density parameters at saddle and in the ground-state (assumed to be a constant). The degeneracy of the four parameter sets is clearly illustrated. However, the application of the same parameter 


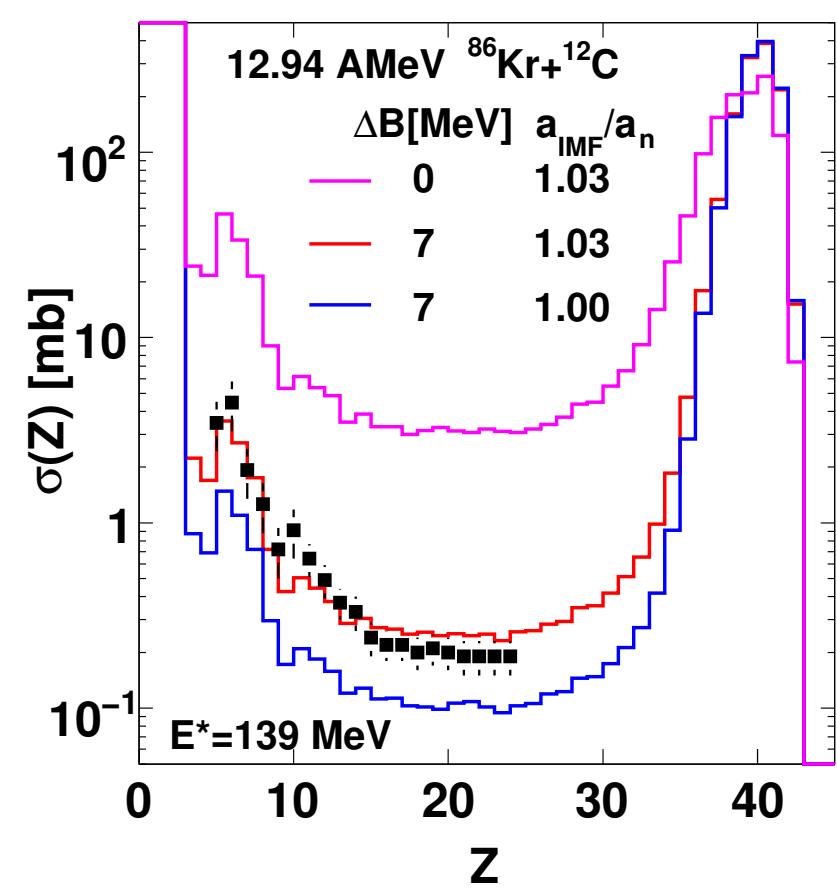

Figure 3. Experimental [19] and calculated residual charge distributions for the indicated fusion reaction.

sets to spallation-fission reactions largely lifts the degeneracy for this observable, as shown in Figure 2 [4].

We now proceed to illustrate the application of the same strategy to fragment charge distributions from non-fissile compound nuclei, which cover IMF-production cross sections. Figure 3 shows the charge distribution of fragments obtained from ${ }^{86} \mathrm{Kr}+{ }^{12} \mathrm{C}$ fusion at $12.94 \mathrm{AMeV}$. The figure illustrates the sensitivity of the calculation results to two parameters: a constant shift of the Sierk IMF barriers $(\triangle B)$ and the saddle-to-ground-state ratio of level-density parameters $\left(a_{\mathrm{IMF}} / a_{\mathrm{n}}\right.$, analogous to the $a_{\mathrm{f}} / a_{\mathrm{n}}$ parameter for fission). IMF yields from fusion show great sensitivity to the barrier height, which is expected because the compoundnucleus nuclear temperature $(T \sim$ a few $\mathrm{MeV})$ is much smaller than the typical IMF barrier height $(B \sim$ a few tens of $\mathrm{MeV}$ ) and the transition rate scales approximately as $\exp (-B / T)$. For the same reason, IMF yields from fusion are relatively insensitive to the small variation of the $a_{\mathrm{IMF}} / a_{\mathrm{n}}$ ratio, which determines the temperature $T$.

In the $1-\mathrm{GeV} \mathrm{p}+{ }^{136} \mathrm{Xe}$ spallation data-set (Figure 4), on the other hand, IMF yields are sensitive to both parameters. This is due to the higher excitation energies that can be reached in spallation: according to the model, fragments with $10<Z<30$ are produced in events with an average excitation energy $\left\langle E^{*}\right\rangle=411 \mathrm{MeV}$, but the distribution extends up to $\sim 750 \mathrm{MeV}$. We can then conclude that, as in the case of fission, combining fusion and spallation datasets allows us to lift some of the degeneracy of the model parameters related to IMF production.

It is tempting to interpret the 7-MeV barrier shift for fusion-IMF cross sections as the difference of Wigner energies between the mother nucleus and the two nascent fragments $[20,21]$. It seems reasonable to assume that the

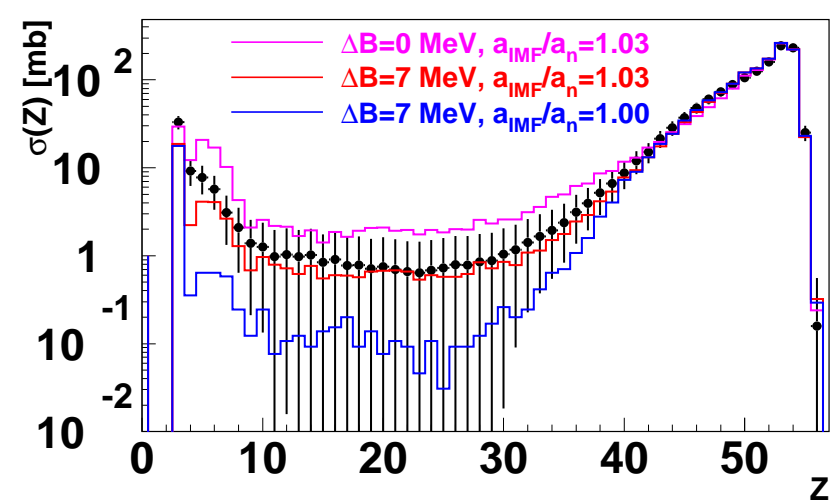

Figure 4. Experimental [27] and calculated residual charge distributions for $1-\mathrm{GeV} \mathrm{p}+{ }^{136} \mathrm{Xe}$.

height of the fission barrier should be sensitive to the Wigner energies of the daughter nuclei only if the saddle-point configuration is sufficiently close to a di-nucleus. This is the case for IMF emission, but not for fission of heavy nuclei; and indeed, we can reproduce fission cross sections of nuclei in the region of $\mathrm{Pb}$ without any barrier shift (Figure 1). However, the Wigner contribution to the binding energy is expected to show some dependence on the asymmetry parameter $I=(N-Z) / A$. The Wigner energy obtained from fitting ground-state masses in the Finite-Range Liquid-Drop Model [22] is

$$
E_{\mathrm{W}}(I)=\left[38.38|I|\left(1-\frac{1}{2} \frac{|I|}{0.35}\right)+2.693\right] \mathrm{MeV}
$$

for $|I|<0.35$. The correction to the barrier for the $\left(Z_{0}, N_{0}\right) \rightarrow$ $\left(Z_{1}, N_{1}\right)+\left(Z_{2}, N_{2}\right)$ process can be expressed as

$$
\Delta B=E_{\mathrm{W}}\left(I_{1}\right)+E_{\mathrm{W}}\left(I_{2}\right)-E_{\mathrm{W}}\left(I_{0}\right) .
$$

Since $N_{1}+N_{2}=N_{0}, Z_{1}+Z_{2}=Z_{0}$, the charge-to-mass ratios of the three nuclei are similar and $E_{\mathrm{W}}(I)$ is approximately linear in $|I|$ for small $|I|$, then $\Delta B \simeq E_{W}\left(I_{0}\right)$ for $N \neq Z$. This suggests to test the interpretation of the barrier shift in terms of Wigner energy by studying compound nuclei of similar size but different asymmetry. However, fitted barrier shifts to IMF yields from ${ }^{70,76} \mathrm{Se}[23],{ }^{75} \mathrm{Br}$ [24], and ${ }^{90,94,98}$ Mo [19] compound nuclei (to be published soon) show that an approximately constant value of $7 \mathrm{MeV}$ is required. Whether this can still be interpreted as a Wignertype contribution is unclear; if Wigner energy is the result of neutron-proton pairing, one might argue that it should fade out with increasing excitation energy, just like pairing between nucleons of the same species. Recent experimental evidence about level densities below and around the particle separation energy indicates that ordinary pairing fades out with excitation energy at about 10-20 MeV $[25,26]$. The compound nuclei we have studied are typically above this value (because a certain amount of kinetic energy is necessary to overcome the fusion barrier); thus, they might be insensitive to the asymmetry dependence of the Wigner energy.

Note incidentally that the parametrisation of the Wigner energy depends of the mass model. Myers and Świątecki's 
Wigner energy applies to masses calculated in the framework of the Thomas-Fermi model [20] and is of the opposite sign to that of Eq. 1. Clearly the sign of the relatively small Wigner term depends on the choice of the mass model and might be different for slightly different surface and volume terms (which are much larger in absolute value than the Wigner term) or different constant terms. Wigner energy should therefore be studied consistently with the choice of the barrier model. In our case we compare the barrier shift to the Wigner energy associated with the Finite-Range Liquid Drop Model calculations of Sierk.

\section{Conclusions}

The fusion and spallation entrance channels probe different regions of the compound-nucleus parameter space and can thus be profitably combined to put stringent constraints on some of the free parameters of de-excitation models. We have demonstrated how this strategy can be fruitfully applied to the study of fission and IMF emission.

In particular, Sierk's finite-range barriers for IMF emission need to be increased by $\sim 7 \mathrm{MeV}$ to fit the data. It is unclear whether this shift can be attributed to the difference between the Wigner energies of mother and daughter nuclei in IMF emission, because the best-fit barriers appear to be independent of the $(N-Z) / A$ ratio of the mother nucleus.

The authors express their gratitude to A. Sierk for the fruitful discussions about the role of Wigner energy in determining fission and IMF barriers.

\section{References}

1. M. Durante, Riv. Nuovo Cimento 25(8), 1 (2002)

2. G. Kraft, Strahlenther. Onkol. 166(1), 10 (1990)

3. M. Salvatores, I. Slessarev, V. Berthou, Progr. Nucl. Energ. 38(1-2), 167 (2001)

4. D. Mancusi, R.J. Charity, J. Cugnon, Phys. Rev. C 82(4), 044610 (2010)

5. C. Schmitt, K.H. Schmidt, A. Kelić, A. Heinz, B. Jurado, P.N. Nadtochy, Phys. Rev. C 81(6), 064602 (2010)

6. R.J. Charity, GEMINI: a code to simulate the decay of a compound nucleus by a series of binary decays, in Joint ICTP-IAEA Advanced Workshop on Model Codes for Spallation Reactions (IAEA, Trieste, Italy, 2008), p. 139, report INDC(NDC)-0530

7. D.J. Hinde, J.R. Leigh, J.O. Newton, W. Galster, S. Sie, Nucl. Phys. A 385, 109 (1982)

8. A.L. Caraley, B.P. Henry, J.P. Lestone, R. Vandenbosch, Phys. Rev. C 62(5), 054612 (2000)

9. A.J. Sierk, Phys. Rev. Lett. 55(6), 582 (1985)

10. N. Carjan, J.M. Alexander, Phys. Rev. C 38(4), 1692 (1988)

11. J.P. Lestone, Phys. Rev. C 59(3), 1540 (1999)
12. A.Y. Rusanov, M.G. Itkis, V.N. Okolovich, Phys. Atom. Nucl. 60(5), 683 (1997)

13. L.G. Moretto, Nucl. Phys. A 247(2), 211 (1975)

14. W. Hauser, H. Feshbach, Phys. Rev. 87(2), 366 (1952)

15. T. Enqvist, W. Wlazło, P. Armbruster, J. Benlliure, M. Bernas, A. Boudard, S. Czajkowski, R. Legrain, S. Leray, B. Mustapha et al., Nucl. Phys. A 686(1-4), 481 (2001)

16. R. Bass, Nucl. Phys. A 231(1), 45 (1974)

17. A. Boudard, J. Cugnon, S. Leray, C. Volant, Phys. Rev. C 66(4), 044615 (2002)

18. J. Cugnon, A. Boudard, S. Leray, D. Mancusi, New features of the INCL4 model for spallation reactions, in International Conference on Nuclear Data for Science and Technology (Korean Nuclear Society and Korean Atomic Energy Research Institute, Jeju Island, Korea, 2010)

19. K.X. Jing, L.G. Moretto, A.C. Veeck, N. Colonna, I. Lhenry, K. Tso, K. Hanold, W. Skulski, Q. Sui, G.J. Wozniak, Nucl. Phys. A 645(2), 203 (1999)

20. W.D. Myers, W.J. Świątecki, Nucl. Phys. A 612(2), 249 (1997)

21. P. Möller, A.J. Sierk, A. Iwamoto, Phys. Rev. Lett. 92(7), 072501 (2004)

22. A.J. Sierk, private communication

23. T.S. Fan, K.X. Jing, L. Phair, K. Tso, M. McMahan, K. Hanold, G.J. Wozniak, L.G. Moretto, Nucl. Phys. A 679(2), 121 (2000)

24. D.N. Delis, Y. Blumenfeld, D.R. Bowman, N. Colonna, K. Hanold, K. Jing, M. Justice, J.C. Meng, G.F. Peaslee, G.J. Wozniak et al., Z. Phys. A 339(2), 279 (1991)

25. M. Guttormsen, R. Chankova, M. Hjorth-Jensen, J. Rekstad, S. Siem, A. Schiller, D.J. Dean, Phys. Rev. C 68(3), 034311 (2003)

26. A.V. Voinov, B.M. Oginni, S.M. Grimes, C.R. Brune, M. Guttormsen, A.C. Larsen, T.N. Massey, A. Schiller, S. Siem, Phys. Rev. C 79(3), 031301(R) (2009)

27. P. Napolitani, K.H. Schmidt, L. Tassan-Got, P. Armbruster, T. Enqvist, A. Heinz, V. Henzl, D. Henzlova, A. Kelić, R. Pleskač et al., Phys. Rev. C 76(6), 064609 (2007) 